REVISTA ANDALUZA DE ANTROPOLOGÍA

NÚMERO 19: DÍAS EXTRAÑOS. CÓMO EL COVID-19 TRANSFORMA NUESTRA COTIDIANIDAD DICIEMBRE DE 2020

ISSN 2174-6796

[pp. 109-116]

https://dx.doi.org/10.12795/RAA.2020.19.06

\title{
MUERTE COLECTIVA Y COVID-19: APUNTES PARA EL DEBATE
}

Jordi Moreras

Universitat Rovira $i$ Virgili

Sol Tarrés

Universidad de Huelva

David Moral

Universidad de Zaragoza

Pilar Gil Tébar

Universidad de Huelva

Ariadna Solé

Universitat Oberta de Catalunya

\section{RESUMEN}

Los efectos de la pandemia han situado a la sociedad española ante una situación de muerte colectiva desconocida desde hace décadas. Además de la saturación de los servicios médicos, el exceso de mortalidad provocó también una situación de colapso de los servicios funerarios. La imposibilidad de llevar a cabo las ceremonias funerarias, o la restricción de participación en las mismas, ha añadido más carga emotiva para aquellas familias que han visto cómo su duelo quedaba suspendido. En este texto aplicaremos la noción de "muerte colectiva" desarrollada por Gaëlle Clavandier, para analizar las acciones conmemorativas llevadas a cabo por las administraciones públicas, en homenaje a las víctimas.

Palabras clave: Muerte; Duelo; Muerte colectiva; Conmemoraciones públicas. 


\begin{abstract}
The effects of the pandemic have plunged Spanish society in a situation of collective death not experienced for decades. In addition to the saturation of medical services, excess mortality also caused a situation of collapse in funeral services. The impossibility of carrying out funeral ceremonies, or the restriction of attendance, has placed an additional emotional burden on families who have had to suspend their mourning. In this article, we will apply Gaëlle Clavandier's notion of "collective death" to analyse the commemorative actions carried out by public administrations, paying tribute to the victims of the pandemic.
\end{abstract}

Keywords: Death; Mourning; Collective death; Public commemorations.

A lo largo de la historia la propagación de enfermedades infecciosas ha generado un desafío a las estructuras sociales en su conjunto, y no solo a los mecanismos de prevención y respuesta sanitaria. Como afirma Snowden (2019), cada situación epidémica ha puesto a prueba a las sociedades que se veían afectadas, evidenciando sus fragilidades. Además, en la comparativa de cómo las pandemias han incidido históricamente en las sociedades, se aprecia una sorprendente regularidad en términos de déficit de prevención, de mecanismos de respuesta eficiente y de caos social generalizado (Spinney, 2018).

Ante la alarma sanitaria provocada por la propagación de la covid-19, todas las instancias sociales se han visto resentidas, generando una alteración de sus ritmos y de sus prácticas, proyectando sobre la vida de los individuos un horizonte inmediato y un futuro condicionado por la incertidumbre. Junto al caos social, se ha producido un episodio de muerte colectiva que no se conocía en Europa - y concretamente en España- desde hace décadas. Al elevado número de fallecidos durante los primeros meses de la pandemia (a fecha de 18 de diciembre de 2020, se ha alcanzado la cifra oficial de 48.926 difuntos), y cuya cuantificación no ha estado exenta de polémica, se le unió el hecho de que un gran número de difuntos (hubieran fallecido o no por coronavirus) no pudieron ser despedidos por sus familiares, ni se pudo celebrar ninguna ceremonia funeraria debido a las restricciones sanitarias que fueron impuestas. También en la muerte la pandemia ha sido excepcional, ya que ha dejado suspendidos muchos duelos familiares.

Todas estas situaciones de excepcionalidad cuestionan la dimensión esencial de servicios que damos por supuestos en tiempos de normalidad. Es el caso de los funerarios. La pandemia ha puesto a prueba la capacidad de respuesta de un sector empresarial constituido en España por unas 1400 empresas, con seis grandes grupos funerarios que 
agrupan casi el $30 \%$ del sector ${ }^{1}$. La normal gestión de sus procesos se vio alterada cuando el gobierno español impuso el estado de alarma el 14 de marzo de 2020 (RD 463/2020), que estableció una serie de medidas sanitarias y de confinamiento social que implicaron, entre otras cosas, la suspensión de todo tipo de velatorios y ceremonias fúnebres. El celo por evitar nuevas propagaciones hizo que algunos ayuntamientos, responsables de esta gestión, interpretaran de forma muy restrictiva este decreto, llegando incluso a prohibir la presencia de familiares en el entierro de sus difuntos. Ante esto, el Ministerio de Salud (Orden SND/298/2020 del 29 de marzo) clarificó esta cuestión al informar que lo que se prohibía eran los velatorios, se posponían las ceremonias fúnebres y se limitaba a tres personas la participación en el ritual funerario de la persona fallecida.

Todo este conjunto de decisiones tuvo un evidente efecto emocional para aquellos que perdieron un allegado durante los primeros meses de pandemia. A la soledad que padecieron los moribundos, se le unió la angustia y el dolor de sus familiares, que no pudieron despedirse de ellos, sin que mediase alguna ceremonia funeraria. $\mathrm{Y}$ a toda esta dimensión emocional, se le añade la saturación de algunos servicios funerarios en los momentos álgidos de incidencia de la covid-19, lo que forzó el uso de espacios alternativos, como el caso paradigmático del Palacio de Hielo de Madrid convertido en morgue provisional. Así pues, los servicios habituales en los tanatorios se limitaron a una dimensión administrativa, y el traslado de los difuntos entre localidades se restringió ${ }^{2}$. El gobierno central también estableció por decreto que los precios de los servicios funerarios no podían ser superiores a los existentes al inicio de la pandemia. Todas estas situaciones han vuelto a dar protagonismo a los cementerios en detrimento de los tanatorios, que en los últimos años se habían convertido en espacios funerarios de referencia.

Esta señalada soledad de los difuntos se vio agravada aún más ante el cruel impacto de la pandemia en las residencias geriátricas españolas ${ }^{3}$, que provocó una alta mortalidad (se calcula que el $67 \%$ de los fallecidos por coronavirus eran mayores que residían en

1. En el número 154 de la Revista Funeraria se elabora un balance de urgencia sobre la respuesta del sector funerario a la crisis provocada por el Covid-19. Disponible en: https://www.revistafuneraria.com/

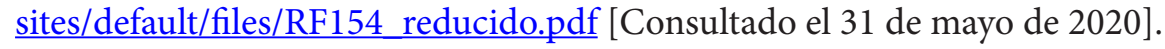

2. En el escenario internacional, el cierre de la frontera marroquí (12 de marzo) supuso frenar de golpe las repatriaciones de los difuntos de origen marroquí que, se calcula, se sitúa en torno al $90 \%$ de los que fallecen en territorio español.

3. Así denominamos nuestro proyecto de investigación: "La soledad de los difuntos. Duelos suspendidos e incertidumbre en tiempos de pandemia". Una primera contribución ya publicada es D. Moral, P. Gil Tébar, A. Solé, S. Tarrés, y J. Moreras, "La soledad de los difuntos", en Stella Evangelidou y Angel Martínez-Hernáez (eds.), RESET: Reflexiones antropológicas ante la pandemia de COVID-19. Tarragona: Publicacions Universitat Rovira i Virgili, 2020, pp. 133-135. 
residencias $)^{4}$. La desastrosa gestión de estos centros durante aquellos meses está siendo discutida mediática y políticamente. Los primeros balances técnicos (Zalakaín y Davey, 2020) muestran la improvisación que caracterizó la atención sanitaria de estas residencias. Pero quizá, lo más destacado es que la pandemia también ha evidenciado que la sociedad se distancia generacionalmente, optando por estos espacios asistenciales como lugares en los que poder gestionar la vejez y las situaciones inmediatamente previas a la muerte. La referencia a la soledad de los moribundos nos recuerda al clásico estudio de Norbert Elias, en el que teorizó que para las sociedades desarrolladas "el proceso de morir se aísla de la vida social normal en un mayor grado que anteriormente" (1987: 139).

Por lo ya planteado, la conciencia de encontrarnos ante una situación de muerte colectiva está reactivando una cierta proximidad con respecto a las circunstancias de la muerte, ya que nos sitúa ante sustantivas cuestiones con respecto a la gestión social del duelo. Para avanzar en esta línea, la noción de muerte colectiva de Gaëlle Clavandier (2004) nos es sumamente útil, por cuanto esta situación de excepcionalidad no solo se define por su número de defunciones (la "muerte en masa"), sino también por la manera cómo esta se ha producido: en el contexto de una pandemia en la que todos nos sentimos potenciales víctimas. Ante ello, nuestra sociedad y sus instituciones respondieron con urgencia para gestionar inicialmente el destino de los cuerpos, racionalizar el luto y, posteriormente, iniciar otra fase de gestión del duelo y de conmemoración colectiva de la memoria de los difuntos.

En este escenario trágico, derivado de la pandemia y el duelo social que esta ha provocado, es muy significativa la voluntad de las instituciones públicas para articular una respuesta que pudiera servir para gestionar el cierre de esos duelos suspendidos. Véase, como ejemplos, el luto oficial decretado por el Gobierno entre el 27 de mayo y el 6 de junio ${ }^{5}$, la ceremonia oficial de homenaje a los fallecidos del 17 de julio en el Palacio Real de Madrid ${ }^{6}$, o tantas otras ceremonias propuestas desde municipios en recuerdo de

4. Disponible en: https://www.rtve.es/noticias/20200915/radiografia-del-coronavirus-residenciasancianos-espana/2011609.shtml [Consultado el 16 de septiembre de 2020].

5. Con una apelación en el preámbulo del decreto que merece ser retenida: "porque es bueno que la sociedad que trabaja junta por el bien común pueda manifestar también junta su dolor, porque es digno consolidar los vínculos sociales con un duelo colectivo y unitario en recuerdo de todas las víctimas provocadas por la violencia, el terror, las catástrofes o la enfermedad" (BOE de 27 de mayo de 2020).

6. Una ceremonia que, finalmente, adquirió una dimensión laica, sin que se realizara ninguna celebración religiosa, y que generó un punto de inflexión con respecto a anteriores funerales de estado. Conociendo la intención del ejecutivo, la Conferencia Episcopal Española convocó una misa funeral el 6 de julio, en la catedral de la Almudena en Madrid, al que asistieron los Reyes. La Vanguardia, 4 de julio de 2020. Disponible en: https://www.lavanguardia.com/politica/20200704/482070018044/obispos-adelantangobierno-convocan-misa-victimas.html [Consultado el 16 de septiembre de 2020]. 
sus paisanos fallecidos. Clavandier (2004), que analizó las respuestas de las instituciones públicas a las catástrofes, nos proporciona algunos elementos para interpretar estas conmemoraciones públicas tras la conmoción social ${ }^{7}$.

Según esta autora, en la respuesta a estas emergencias se suceden cuatro momentos: una primera fase de conmoción e impacto ante lo sucedido (en este caso, ante lo que todavía está sucediendo) que abruma y descoloca; esta es seguida por otra segunda fase de racionalización en la que impera la gestión burocrática de la respuesta respecto lo sucedido. Este momento es crítico para la acción política de las instituciones. Así, las primeras iniciativas suponen activar todos aquellos recursos disponibles que, siendo apropiados a una situación de normalidad, se ven desbordados en este escenario excepcional. En esta segunda fase, se concentra una parte muy importante del debate político y social, en el que se juzga la capacidad y madurez de los equipos de gobierno para responder a lo sucedido, en un tono áspero y violento, acorde con la tragedia. Es un terreno abonado para el oportunismo político, para el desmarque y para cosechar la sombra de duda y sospecha, puesto que se cuestiona tanto su incapacidad para prevenir y anticiparse a lo sucedido, como la idoneidad y oportunidad de las inmediatas decisiones adoptadas.

Tras esta fase de cacofonía de acusaciones que reclaman responsabilidades a las instituciones públicas, y que interfieren en su gestión de urgencia, se abre un tercer momento en el que se articula un mecanismo para poder aplacar todo este ruido político y mediático. Y la forma de hacerlo es apelar al silencio que acompaña toda sentida conmemoración de lo ocurrido y a pronunciar el recuerdo de las víctimas. Y es así como esta apelación a la remembranza de las víctimas y de homenaje a los trabajadores esenciales, ha servido para acallar, aunque sea temporalmente, la suma de críticas y sospechas. Es aquí donde se sitúan todo este conjunto de ceremonias públicas que se han llevado a cabo en los últimos meses, y que pretenden dar testimonio de lo sucedido.

Hemos denominado genéricamente a estos actos como "expresiones performativas del duelo", puesto que se proyectan en el espacio público, superando esa supuesta dimensión de recogimiento íntimo o familiar que acompaña todo duelo ${ }^{8}$. En este sentido, lo que se articula a través de estas conmemoraciones va más allá del recuerdo de los difuntos, o del reconocimiento de los servicios esenciales, pues contienen un implícito contenido

7. Véase Jordi Moreras y David Moral, "De la conmoción a la conmemoración", en Sociología en cuarentena, 22 de julio de 2020. Disponible en: https://sociologiaencuarentena.tumblr.com/ post/624328007446822912/de-la-conmoci\%C3\%B3n-a-la-conmemoraci\%C3\%B3n [Consultado el 16 de septiembre de 2020].

8. Seguimos la propuesta de Paul Connerton (1989), de atender a las dimensiones performativas de los actos de apropiación institucional de la memoria colectiva. 
político. Como en su tiempo ya nos recordó La Rochefoucauld, la pompa de las ceremonias fúnebres tiene que ver más con la vanidad de los vivos que con el homenaje a los difuntos; o lo que es lo mismo: estos actos se piensan más para los vivos que para los muertos.

Por lo tanto, mezclar en un mismo acto recuerdo y homenaje podría parecer paradójico, si no fuera porque estas ceremonias pretenden también rendir cuentas respecto lo sucedido. Esto se observa en las conmemoraciones celebradas por los consistorios municipales, en los que la referencia a la gestión de la pandemia en las residencias geriátricas ha sido una constante. El recuerdo explícito de lo sucedido se convierte en el primer paso de cara a superarlo y, por lo tanto, tiene sentido homenajear a aquellos que se dejaron la piel cuidando a otros, y lo siguen haciendo, aunque ya con un reconocimiento diverso.

Además, y acorde con lo que ha sido una constante durante estos meses, estos actos también se convierten en momentos para proyectar un mensaje de esperanza de cara al futuro, asegurando que como sociedad saldremos reforzados de esta crisis. De ahí la articulación de simbologías en la escenografía de estos actos, como el dejar el protagonismo en los parlamentos a los familiares y trabajadores esenciales, y el respetuoso silencio como bálsamo ante la crítica política y social. Estas ceremonias adquieren una dimensión catártica, anunciando la voluntad de seguir adelante, y haciendo una llamada a la unidad de la sociedad (que, implícitamente, no deja de ser una apelación para recuperar la confianza en las instituciones). Estas ceremonias, finalmente, también se pensaron como forma de establecer un primer cierre temporal respecto a lo sucedido, $y$ marcan el inicio de lo que ha enunciado como "nueva normalidad", una entelequia sin concreción, pero un marco de referencia fundamental para impulsar el ejercicio de la política en estos tiempos de incertidumbre (Innerarity, 2020).

Sin embargo, todos estos ejercicios de cierre categorial han quedado en evidencia ante el resurgir de la pandemia y el advenimiento de nuevos brotes comunitarios tras el verano y las fiestas navideñas. De ahí que no haya que descartar que en un futuro inmediato se vuelvan a reproducir. Porque lo relevante de estas conmemoraciones es que abren el camino al cuarto y último momento, que en términos oficiales se describe como de memoria. Clavandier explica que los gobiernos no pueden mantener permanentemente el recuerdo de lo sucedido, pues es necesario re-construir la normalidad y cerrar progresivamente el duelo social que provocó la tragedia.

En este sentido, la muerte colectiva no puede estar contaminando permanentemente la vida social. De ahí que se sugieran iniciativas para fijar la memoria, tanto espacial como temporalmente. En la primera dimensión ya se han sugerido algunas iniciativas, para emplazar algún monumento conmemorativo en cementerios o lugares públicos. Pero 
la segunda es mucho más difícil de establecer, puesto que no es sencillo concretar la efeméride de la pandemia: ¿será el día en que falleció la primera persona por la covid-19, el homenaje del 16 de julio, o cualquier otra fecha??.

Como bien sabemos, las ceremonias conmemorativas sirven principalmente como ejercicio de reconstrucción oficial de lo sucedido y de cómo debe ser establecida esa memoria colectiva que, como bien dijo Maurice Halbwachs, parte del presente para reconstruir el pasado, y que ayuda a que la sociedad aleje de su memoria aquello que pueda servir para separar a los individuos. Todo este proceso de apropiación desde el poder de cualquier otra forma de memoria colectiva debe ser analizado y puesto en contraste con respecto a la memoria social, que es retenida y reflejada por aquellos que han tenido la experiencia del dolor vivido ante la pérdida de sus allegados ${ }^{10}$, en este caso agravado por el hecho de siquiera haberse podido despedir de ellos. Se está "normalizando" una situación excepcional de muerte, todavía quedan duelos que siguen suspendidos y no habrá ceremonias con cuidada escenografía o hasthags pegadizos que puedan resolverlos.

En definitiva, la pandemia ha puesto de relieve algunas de las ambigüedades y contradicciones que nuestra sociedad mantiene con respecto a la vida y a la muerte, así como ha señalado a todas aquellas construcciones sociales que hemos elaborado al respecto. Tras la pandemia, sin duda recuperaremos una "normalidad" que deberá ser construida ad hoc para los tiempos venideros. La contribución de la antropología debería consistir en analizar lo sucedido, etnografiar los diferentes terrenos en los que se ha desplegado la respuesta al periodo excepcional que seguimos viviendo, y valorar - de acuerdo con nuestro enfoque específico- la fuerza reparadora de los rituales sociales que hemos elaborado en torno a la muerte, y de las conmemoraciones sociales con las que queremos elaborar nuestra memoria colectiva.

9. De hecho, esta decisión siempre será política. Véase como ejemplo, en el anteproyecto de ley de la Memoria democrática, cómo se establece el día 31 de octubre como el día de recuerdo y homenaje a todas las víctimas de la Guerra Civil y la Dictadura franquista. Disponible en: https://www.lamoncloa.gob.es/ consejodeministros/referencias/documents/2020/refc20200915.pdf [Consultado el 16 de septiembre de 2020].

10. Esta forma de memorialización del duelo colectivo y de la retención de una memoria social, puede observarse en el trabajo de Cristina Sánchez Carretero (2011), en relación con los atentados terroristas del 11-M de 2004 en Madrid. 


\section{REFERENCIAS BIBLIOGRÁFICAS}

Clavandier, Gaëlle (2004) La mort collective. Pour une sociologie des catastrophes. París: CNRS Éditions.

Connerton, Paul (1989) How societies remember. Londres: Cambridge University Press. Elias, Norbert (1987) La soledad de los moribundos. México: Fondo de Cultura Económica. Halbwachs, Maurice (1950) La mémoire collective. París: Presses Universitaires de la France.

Innerarity, Daniel (2020) Pandemocracia. Una filosofía de la crisis del coronavirus. Barcelona: Galaxia Gutenberg.

Sánchez Carretero, Cristina (coord.) (2011) El archivo del duelo. Análisis de la respuesta ciudadana ante los atentados del 11 de marzo en Madrid. Madrid: Centro Superior de Investigaciones Científicas.

Snowden, Frank M. (2019) Epidemics and society. From the black death to the present. New Haven-Londres: Yale University Press.

Spinney, Laura (2019) El jinete pálido. 1918: la epidemia que cambió el mundo. Barcelona: Crítica.

Zalakaín, Joseba, y Vanessa Davey (2020) "The covid-19 on users of long-term care services in Spain". International long term care policy network. Disponible en: https:// ltccovid.org/wp-content/uploads/2020/05/LTCcovid-Spain-country-report-28-May-1. pdf [Consultado: 7 de octubre de 2020]. 